\title{
Cholesterol reduction using manufactured foods high in monounsaturated fatty acids: a randomized crossover study
}

\author{
C. M. Williams ${ }^{1 *}$, J. A. Francis-Knapper ${ }^{2}$, D. $\mathrm{Webb}^{2}$, C. A. Brookes ${ }^{2}$, A. Zampelas ${ }^{2}$, J. A. Tredger ${ }^{2}$, \\ J. Wright ${ }^{2}$, G. Meijer ${ }^{3}$, P. C. Calder ${ }^{4}$, P. Yaqoob ${ }^{4}$, H. Roche ${ }^{5}$ and M. J. Gibney ${ }^{5}$ \\ ${ }^{1}$ Hugh Sinclair Unit of Human Nutrition, Department of Food Science and Technology, University of Reading, \\ Reading RG6 6AP, UK \\ ${ }^{2}$ School of Biological Sciences, University of Surrey, Guildford GU2 5XH, UK \\ ${ }^{3}$ Unilever Research Laboratory, Vlaardingen, The Netherlands \\ ${ }^{4}$ Department of Biochemistry, University of Oxford, Oxford OX1 3QU, UK \\ ${ }^{5}$ Department of Medicine, Trinity College, Dublin, Republic of Ireland
}

(Received 24 February 1998 - Revised 2 December 1998 - Accepted 15 December 1998)

\begin{abstract}
In two separate studies, the cholesterol-lowering efficacy of a diet high in monounsaturated fatty acids (MUFA) was evaluated by means of a randomized crossover trial. In both studies subjects were randomized to receive either a high-MUFA diet or the control diet first, which they followed for a period of 8 weeks; following a washout period of 4-6 weeks they were transferred onto the opposing diet for a further period of 8 weeks. In one study subjects were healthy middle-aged men $(n 30)$, and in the other they were young men $(n 23)$ with a family history of CHD recruited from two centres (Guildford and Dublin). The two studies were conducted over the same time period using identical foods and study designs. Subjects consumed 38\% energy as fat, with $18 \%$ energy as MUFA and $10 \%$ as saturated fatty acids (MUFA diet), or $13 \%$ energy as MUFA and $16 \%$ as saturated fatty acids (control diet). The polyunsaturated fatty acid content of each diet was $7 \%$. The diets were achieved by providing subjects with manufactured foods such as spreads, 'ready meals', biscuits, puddings and breads, which, apart from their fatty acid compositions, were identical for both diets. Subjects were blind to which of the diets they were following on both arms of the study. Weight changes on the diets were less than $1 \mathrm{~kg}$. In the groups combined ( $n$ 53) mean total and LDL-cholesterol levels were significantly lower at the end of the MUFA diet than the control diet by $0.29(\mathrm{SD} 0.61) \mathrm{mmol} / \mathrm{l}(P<0.001)$ and $0.38(\mathrm{SD} 0.64) \mathrm{mmol} / \mathrm{l}(P<$ $0.0001)$ respectively. In middle-aged men these differences were due to a mean reduction in LDL-cholesterol of -11 (SD 12) \% on the MUFA diet with no change on the control diet $(-1 \cdot 1$ (SD 10) \%). In young men the differences were due to an increase in LDL-cholesterol concentration on the control diet of $+6 \cdot 2$ (SD 13) $\%$ and a decrease on the MUFA diet of $-7 \cdot 8$ (SD 20) $\%$. Differences in the responses of middle-aged and young men to the two diets did not appear to be due to differences in their habitual baseline diets which were generally similar, but appeared to reflect the lower baseline cholesterol concentrations in the younger men. There was a moderately strong and statistically significant inverse correlation between the change in LDLcholesterol concentration on each diet and the baseline fasting LDL-cholesterol concentration $(r-0.49 ; P<0.0005)$. In conclusion, diets in which saturated fat is partially replaced by MUFA can achieve significant reductions in total and LDL-cholesterol concentrations, even when total fat and energy intakes are maintained. The dietary approach used to alter fatty acid intakes would be appropriate for achieving reductions in saturated fat intakes in whole populations.
\end{abstract}

Coronary heart disease: Monounsaturated fatty acids: Plasma cholesterol

CHD is the major cause of premature death and morbidity in the UK, with Scotland having the highest rate of CHD in the world. Recent primary and secondary drug trials have demonstrated the clear benefit of plasma cholesterol reduction in both the prevention and treatment of CHD (Scandinavian Simvastatin Survival Group, 1994; Shepherd et al. 1995) and re-emphasize the importance of public health strategies aimed at reducing plasma cholesterol 
levels. Drug treatment has been shown to reduce CHD mortality by $33 \%$ in subjects with levels of plasma cholesterol in the upper end of the normal range (Shepherd et al. 1995). These represent individuals who would not normally be considered candidates for pharmacological intervention and who represent approximately $30 \%$ of the middleaged UK population. Effective dietary strategies aimed at reducing plasma cholesterol concentrations in middle-aged subjects with moderately raised cholesterol levels, and at preventing age-associated increases in plasma cholesterol in younger age groups, have important public health implications.

Current dietary recommendations for prevention of CHD in the UK population focus on the need for reductions in total fat and especially saturated fatty acids (SFA) (notably lauric, myristic and palmitic acids), since these have been shown to raise plasma cholesterol levels in controlled feeding studies. Expert bodies advise that SFA should decrease from the current level of intake of $16 \%$ to $10 \%$ of dietary energy (Department of Health, 1994; Pyörälä et al. 1994). Specific recommendations for changes in the intakes of the other major fatty acid classes, monounsaturated fatty acids (MUFA) and polyunsaturated fatty acids (PUFA), were not made in the UK Department of Health (1994) report, because of continuing debate regarding the overall benefits of additional intakes of these fatty acids.

Since the publication of UK national guidelines for fat intake in 1991 (Department of Health, 1991), and their re-iteration in 1994 (Department of Health, 1994), there has been some movement towards achievement of population target intakes for total fat and SFA, but levels remain considerably higher than desired target values (Ministry of Agriculture, Fisheries and Food, 1992). This may be due, in part, to lack of consumer preference for low-fat products and low adherence, in the long term, to fat-reduced commodities.

In recent years we have had the opportunity to investigate the effects of increasing dietary MUFA intake in two separate study populations. These were (1) a group of young men from the UK and Republic of Ireland with a family history of CHD and (2) a group of normal middleaged men from the UK. The groups were studied as part of two intervention studies which evaluated effects of a highMUFA diet on a range of outcome markers including postprandial lipoproteins, blood coagulation factors and immune function. Some of the data from these studies have been reported in detail elsewhere (Roche et al. 1998; Yaqoob et al. 1998). Details of the diet itself and the findings for fasting blood lipid variables have not previously been reported in detail. Since the diets used in the two studies were identical, and the studies were carried out over the same time period, we have made a comparison of the effects of the MUFA diet on fasting blood lipids in young and middle-aged men who represent subjects with a range of fasting blood lipid values.

In each study a randomized crossover design was used. Two diets were compared, one high in SFA, which had a fatty acid profile similar to current UK fatty acid intakes, and one high in MUFA, which had a fatty acid profile similar to current Mediterranean intakes. In the high-MUFA diet, MUFA were substituted for SFA in an isoenergetic manner in order to achieve the target intakes of MUFA (18\% energy) and SFA (10\% energy); total fat intake was maintained at current levels of intake (38\% energy) and intakes of other major fatty acids were maintained constant on the two diets. Although the fatty acid profile of the MUFA diet used in the present study was close to that of Mediterranean populations, it was considered important to provide foods and meals which reflected the food culture of the UK, which it would be feasible to implement widely in the UK and Republic of Ireland. A particular feature of the study was that, in addition to providing subjects with modified cooking fats and spreads we also provided them with pre-prepared manufactured recipe meals, biscuits and puddings which were identical to current commercially available products, except that they were manufactured using the experimental oils and spreads. The aim of this approach was to determine the feasibility of achieving the dietary fat target intakes using 'supermarket' foods commonly eaten in the UK and Republic of Ireland.

\section{Methods}

Thirty healthy middle-aged men and thirteen healthy younger men were recruited through general practitioners in the Guildford area and ten healthy younger men were also recruited from a second centre in Dublin. The young men were in good health, but each had a first-degree relative with CHD (defined as either a myocardial infarct, angioplasty or coronary artery bypass surgery). All volunteers were in general good health as assessed by medical history, were non-smokers, not vegetarian, drank less than 30 units of alcohol per week (confirmed by the assessment). Subjects' habitual diets provided 35-45\% energy as fat; no subjects were taking either a dietary supplement or any medication known to affect carbohydrate or lipid metabolism. Subjects abstained from exercise and alcohol consumption for $24 \mathrm{~h}$ before giving a fasted blood sample for screening purposes and before all subsequent follow-up visits. The biochemical exclusion limits were one or more of the following: a $12 \mathrm{~h}$ fasting total plasma cholesterol level of $>6.5 \mathrm{mmol} / \mathrm{l}$; a plasma triacylglycerol concentration of $>2.5 \mathrm{mmol} / \mathrm{l}$; a blood glucose level of $>6.2 \mathrm{mmol} / \mathrm{l}$ or a haemoglobin level of $<130 \mathrm{~g} / \mathrm{l}$.

The study was undertaken after approval from the University of Surrey, the Royal Surrey County Hospital and Federated Dublin Voluntary Hospitals in Ireland Ethics Committees; subjects gave their informed written consent to participate.

Subjects were assigned to receive two diets (control and MUFA) in a randomized single-blind crossover design of 6 months total duration in a free-living situation. Each diet period lasted 2 months with a washout period of approximately 6 weeks between each arm of the study. Following initial baseline assessments subjects returned to the study centres at $0,2,4,6$ and 8 weeks to collect food and for further anthropometric and biochemical measurements. Subjects were provided with foods containing the experimental fats and given advice on meal preparation, eating out and snacks; foods provided by the investigators accounted for approximately $60 \%$ of subjects' total daily fat intake. 
If desired, foods were also provided for subjects' partners to aid food preparation in the home and to optimize compliance.

\section{The diet}

The aim of the control diet was to achieve fat and fatty acid intakes equivalent to those of the current UK intake (Gregory et al. 1990) (\% dietary energy: 38 total fat, 16 SFA, 12 MUFA, 6 PUFA); the aim of the MUFA diet was to replace $40 \%$ of the SFA with MUFA (\% dietary energy: 38 total fat, 10 SFA, 18 MUFA, 6 PUFA). Foods were produced in collaboration with fats and oils producers, food manufacturers and retailers. Test (MUFA) and control (SFA) blends of cooking fats and spreads were made by Van den Bergh Foods, Crawley, Sussex, UK. The MUFA cooking fat was $100 \%$ fat, comprising ( $\mathrm{g} / 100 \mathrm{~g}$ total fatty acids): $16: 0,12 ; 18: 0,6 ; 18: 1,59 ; 18: 2,21$; other fatty acids, 2 . The SFA fat comprised $(\mathrm{g} / 100 \mathrm{~g}$ total fatty acids): $16: 0$, $28 \cdot 5 ; 18: 0,13 ; 18: 1,34 ; 18: 2,19$; other fatty acids, $4 \cdot 5$. The spreads were prepared from the same fat blends and were $80 \%$ fat. The desired fatty acid compositions of the spreads and fat blends were calculated from details of diet diaries recorded by fifteen subjects some months before the intervention study and on the basis of a pilot study conducted 12 months previously. The fatty acid compositions of the non-intervention foods, which represented nonexchangeable fats, were estimated. The fatty acid compositions of exchangeable fats and foods prepared from them (intervention foods), were estimated as the difference between non-intervention food and total diet target fatty acid intakes. Vitamins were added at usual levels and salt added to levels acceptable to the UK palate. These cooking fats and spreads were used domestically by the subjects and additionally were provided to Health and Diet Biscuits Ltd, Sheffield, UK and Sainsburys plc, UK, to replace the usual extrinsic fat in a range of biscuits (four varieties), recipe meals (eight varieties), puddings (two varieties) and garlic bread. Foods that were identical apart from fatty acid composition were provided for control and MUFA arms of the study. In addition, commercially available low-fat recipe meals (ten varieties), potatoes for jacket potato, stirfry vegetables and rice were used to offer more food choices and subjects were able to add the cooking fats and spreads to these. To achieve the target fatty acid intakes subjects were required to consume between 30 and $55 \mathrm{~g}$ MUFA or SFA per diet day depending on their habitual fat intake. All portions of food were designed to contain multiples of $5 \mathrm{~g}$ MUFA or SFA (referred to as a lipid unit). Spreads for domestic use were provided in standard $250 \mathrm{~g}$ packs but subjects were given a standard serving measure which represented one lipid unit. The number of lipid units contained within a single portion of a food ranged from 1 to 4 and was labelled on the packaging (examples shown in Table 1). Thus subjects consumed between 6 and 11 lipid units per diet day by choosing foods they liked each day to their lipid unit target. Subjects were asked to eat the recipe meals provided on $5 \mathrm{~d}$ each week and on $2 \mathrm{~d}$ they were allowed to provide their own meals and/or to eat out. Spreads, cooking fats and biscuits were eaten every day. Intervention spreads and food accounted for approximately two-thirds of the total fat intake of the subjects, MUFA or
Table 1. Examples of the lipid unit content of intervention foods

\begin{tabular}{lc}
\hline Foods & Lipid units \\
\hline Meat lasagne & 1 \\
Shepherd's pie & 4 \\
Seafood tagliatelle & 3 \\
Vegetable curry & 2 \\
Beef cobbler & 2 \\
Garlic bread (two slices) & 1 \\
Sticky toffee pudding & 3 \\
Spread (10g scoop) & 1 \\
Biscuits (4) & 1 \\
\hline
\end{tabular}

SFA accounted for approximately $40 \%$ total daily fat intake on each of the diets. The remaining 'non-exchangeable' fat in the diet came from cheese and dairy products (milk, cream, ice-cream, yoghurts) whose fatty acid compositions could not be altered and from the 'free meal' periods (two per week) when subjects were allowed to eat foods other than those supplied by the investigators. Although subjects were guided in the amounts of the types of foods which were allowable, these 'free meal' periods were found to be of importance in allowing subjects a degree of control over their own meals, and aided compliance.

\section{Dietary assessment}

An unstructured diet diary was completed by each subject for a $5 \mathrm{~d}$ period during weeks 3 and 7 of each arm of the study. Each $5 \mathrm{~d}$ diary covered four diet days and one free day. All fats and foods prepared for the study were analysed for their nutrient and fatty acid compositions by Reading Scientific Services Ltd, Reading, Berks., UK. This information, along with the portion sizes of the experimental meals, biscuits, cakes and puddings, was added to the Foodbase database of UK food tables (Institute of Brain Chemistry, London, UK). Diet diaries were analysed for nutrient composition using Foodbase. Amounts of food consumed were quantified using household measures, standard food portion sizes and weights of foods provided in manufacturer's information.

\section{Dietary compliance}

This was self-assessed using a questionnaire incorporating a linear-analogue scale. Subjects completed this questionnaire after 4 and 8 weeks on each arm of the study.

\section{Anthropometric and biochemical measurements}

Subjects were weighed once fortnightly by the investigators. All subjects weighed themselves daily for the first fortnight on each arm of the study and then once weekly thereafter. The number of lipid units was adjusted if subjects gained or lost more than $2 \mathrm{~kg}$ over a 2 -week period. Blood samples were collected before (0) and at 2, 4 and 8 weeks within each arm of the study. Subjects attended the investigation unit after an overnight fast. On these occasions a $10 \mathrm{ml}$ blood sample was taken. Plasma was separated immediately and portions were frozen to $-20^{\circ}$ for later analyses. 
Table 2. Anthropometric, blood lipid and dietary fat characteristics of young (UK and Irish) and middle-aged (UK only) subjects at baseline (Mean values and standard deviations)

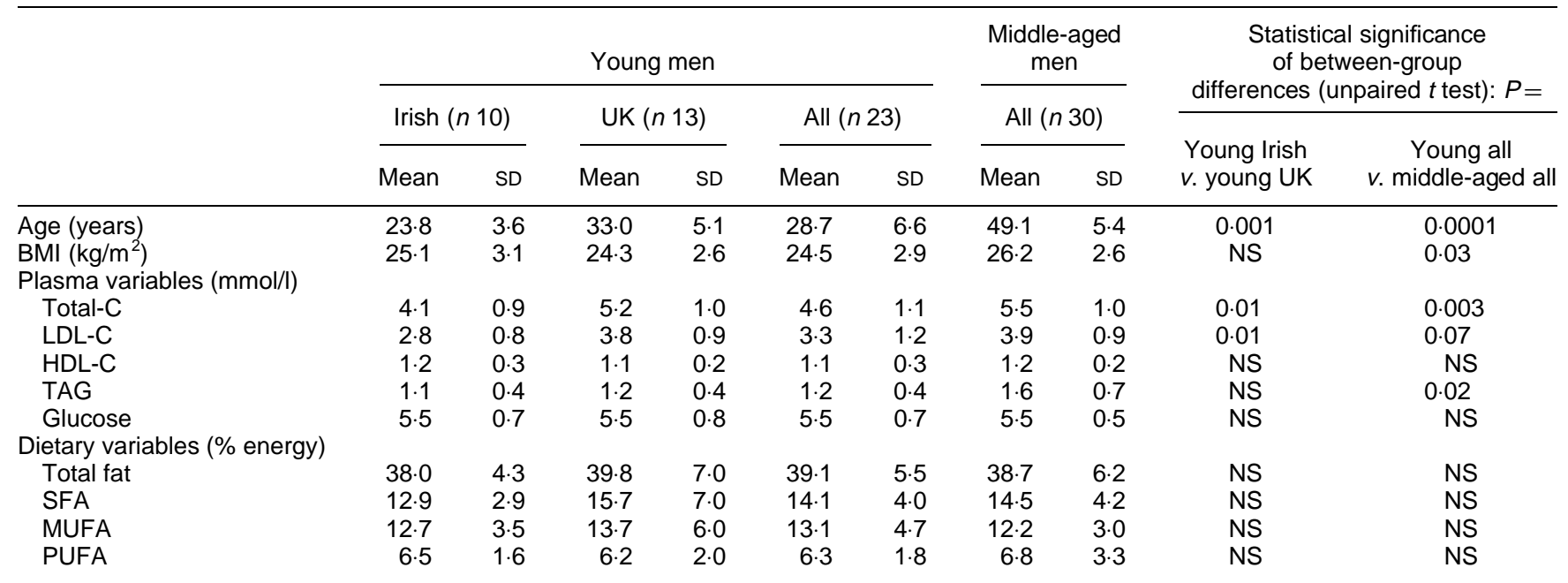

Total-C, total cholesterol; LDL-C, LDL-cholesterol; HDL-C, HDL-cholesterol; TAG, triacylglycerol; SFA, saturated fatty acids; MUFA, monounsaturated fatty acids; PUFA, polyunsaturated fatty acids.

\section{Analytical methods}

Blood samples were centrifuged at $1700 \mathrm{~g}$ immediately after collection to separate plasma. Enzymic colorimetric methods were used on a Cobas-Mira analyser (Roche Products Ltd, Basel, Switzerland) to measure glucose, cholesterol (Cholesterol PAP Uni-kit; Roche Products Ltd), triacylglycerol (TAG PAP Uni-kit; Roche Products Ltd) and non-esterified fatty acids (Wako NEFA-C test kit; Alpha Laboratories Ltd, Eastleigh, Hants., UK). HDLcholesterol levels were determined in fresh plasma following precipitation of LDL and VLDL (Immuno Ltd, Sevenoaks, Kent, UK). Following precipitation the supernatant fraction was removed and stored at $-20^{\circ}$ until analysis. The interassay $\mathrm{CV}$ for these methods was $<5 \%$. LDLcholesterol levels were calculated from Friedewald's formula (Friedewald et al. 1972) where LDL-cholesterol = total cholesterol - HDL-cholesterol - triacylglycerol/2.2, all values expressed as $\mathrm{mmol} / \mathrm{l}$.

In thirteen of the young men plasma triacylglycerol fatty acid composition was analysed at the end of the control and MUFA diet periods as previously described (Roche et al. 1998). In twenty-two middle-aged men plasma phospholipid fatty acid composition was analysed at the end of the control and MUFA diet periods as previously described (Yaqoob et al. 1998). These data were used to provide an objective index of dietary compliance.

\section{Data handling and statistics}

Results are expressed as means with their standard errors in figures and means and standard deviations in tables. Statistical analysis was performed using Data Desk 4.1 (Data Descriptions Inc., Ithaca, NY, USA). Data were analysed by diet and age group and by time. Normal distribution of the data was achieved by transformation as appropriate. $P<0.05$ was accepted as the level of statistical significance.

\section{Results}

Young Irish men were significantly younger than young UK men (Table 2; $P<0 \cdot 001$ ), and as expected, the young men as a whole were significantly younger than the middle-aged men $(P<0.0001)$. Middle-aged men had a higher BMI than young men $(P<0.03)$. Young Irish men had lower fasting total and LDL-cholesterol levels $(P<0 \cdot 01)$ than young UK men and measurements of plasma total cholesterol and triacylglycerol levels were higher in the middle-aged men than the young men (all) $(P<0.003$ and $P<0.02$ respectively) (Table 2). Habitual diets did not differ between either young Irish and young UK men, or between young men (all) and middle-aged men. Although there was a trend for intakes of dietary SFA to be lower in young Irish men than both UK groups this difference did not reach a level of statistical significance.

The dietary intervention successfully achieved the target fatty acid intakes, with mean levels of intake for the group as a whole ( $n$ 53) of (\% energy): 15.9 (SD 1.5) SFA and 12.9 (SD 1.5) MUFA on the control diet and 10.1 (SD 1.7) SFA and 17.7 (SD 1.9) MUFA on the MUFA diet (combined data not shown in tables). For both young and middle-aged men the only differences between control and MUFA diets were the intakes of SFA and MUFA which were significantly different between the two diets for both age groups (Table 3; $P<0.0001$ in all cases). Percentages of energy as total fat, PUFA, carbohydrate and protein did not differ between the two diets. Comparison of nutrient intakes in young and middle-aged men (Table 3) showed that there were more differences between the two age groups for the MUFA diet than for the control diet. On the MUFA diet young men consumed significantly less energy as protein $(P<0 \cdot 01)$ and carbohydrate $(P<0.05)$ and more energy as total fat $(P<0.0001)$, SFA $(P<0.0001)$, MUFA $(P<0.01)$ and PUFA $(P<0.004)$ than did middle-aged men on the same diet. During the control diet period young men obtained 
Table 3. Mean daily intakes of energy and percentages of energy as protein, carbohydrate and fat and the major fatty acid classes for young and middle-aged subjects on control and monounsaturated fatty acid (MUFA)-enriched diets*

(Mean values and standard deviations)

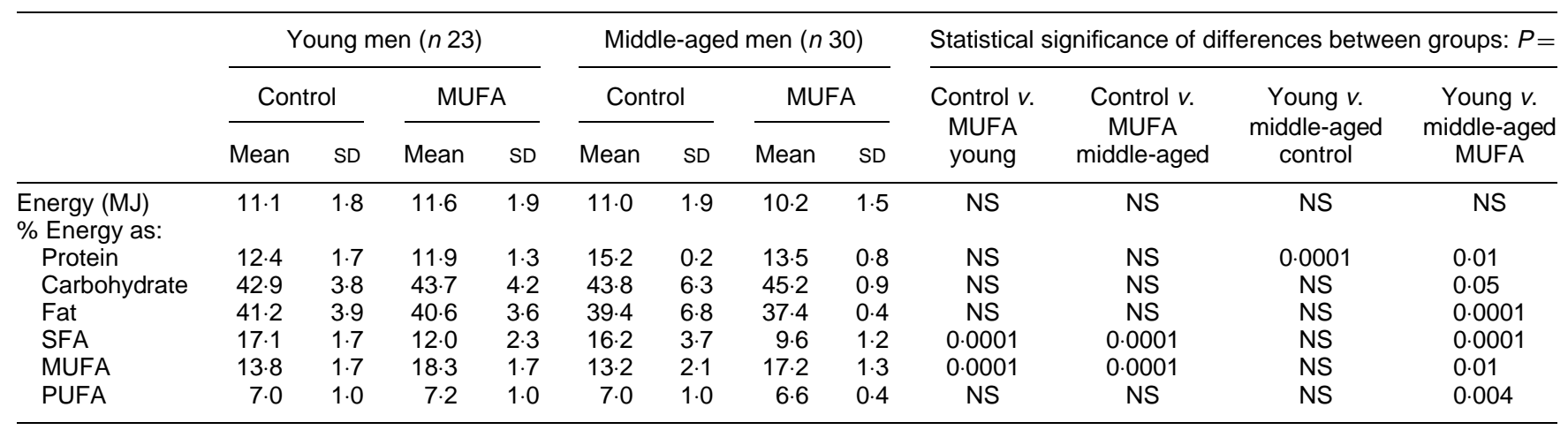

SFA, saturated fatty acids; PUFA, polyunsaturated fatty acids.

${ }^{*}$ For details of subjects and diets, see Table 2 and pp. 440-442.

significantly more energy from protein than did middleaged men $(P<0 \cdot 0001)$, but other differences did not reach levels of statistical significance.

Some assessment of the degree of compliance with the diet was provided by measurement of fasting plasma triacylglycerol fatty acid composition in a cohort of the young men and by plasma phospholipid fatty acid composition in a cohort of the middle-aged men (Table 4). In young men there was a significantly higher plasma triacylglycerol MUFA content at the end of the MUFA diet compared with the end of the control diet $(P<0 \cdot 002)$. There was a trend for plasma triacylglycerol SFA content to be reduced on the MUFA diet, but this did not reach a level of statistical significance $(P<0.07)$. Similarly in middle-aged men, plasma phospholipid MUFA content was higher at the end of the MUFA diet than the control diet $(P<0.0001)$ and there was an opposite trend in the SFA content but the latter did not reach a level of statistical significance. PUFA content did not show any change in any of the plasma lipids for either of the age groups on either of the two diets.

Table 5 shows the mean values for fasting blood lipids for the group as a whole at weeks $0,2,4$ and 8 of the control and MUFA diets. Total and LDL-cholesterol levels were significantly lower at the end of the MUFA diet than the control diet $(P<0.0001$ and $P<0.001$ respectively). During the control diet there were no significant changes from baseline to week 8 in any of the fasting lipid values. During the MUFA diet there were significant reductions in fasting total and LDL-cholesterol levels between baseline and week 8 $(P<0.005$ and $P<0.001$ respectively). There were no significant differences in HDL-cholesterol, triacylglycerol or glucose concentrations between the two diets in either group. There were no significant differences in body weight at the end of the control and MUFA diets. However, during each 8-week diet period there was a small increase in body weight of approximately $1 \mathrm{~kg}$ on both control and MUFA diets $(P<0.05$ both diets).

The mean difference for the end of the control diet compared with the end of the MUFA diet for total cholesterol was 0.29 (SD 0.61$) \mathrm{mmol} / \mathrm{l}(P<0.001)$ and for LDLcholesterol was 0.38 (SD 0.64$) \mathrm{mmol} / \mathrm{l} \quad(P<0.0001)$. Differences were found in the nature of the responses of young men and middle-aged men to the control and MUFA diets (Fig. 1). Middle-aged men showed a mean reduction of -11 (SD 12) \% in LDL-cholesterol from baseline on the MUFA diet, with no change in response to the control diet $(-1 \cdot 1$ (SD 10$) \%)$. In contrast young men showed an increase in LDL-cholesterol on the control diet of $+6 \cdot 2$

Table 4. Values for fasting plasma phospholipid fatty acid composition (middle-aged men) and plasma triacylglycerol fatty acid composition (young men) at the end of control and monounsaturated fatty acid (MUFA)-enriched diets*

(Mean values and standard deviations)

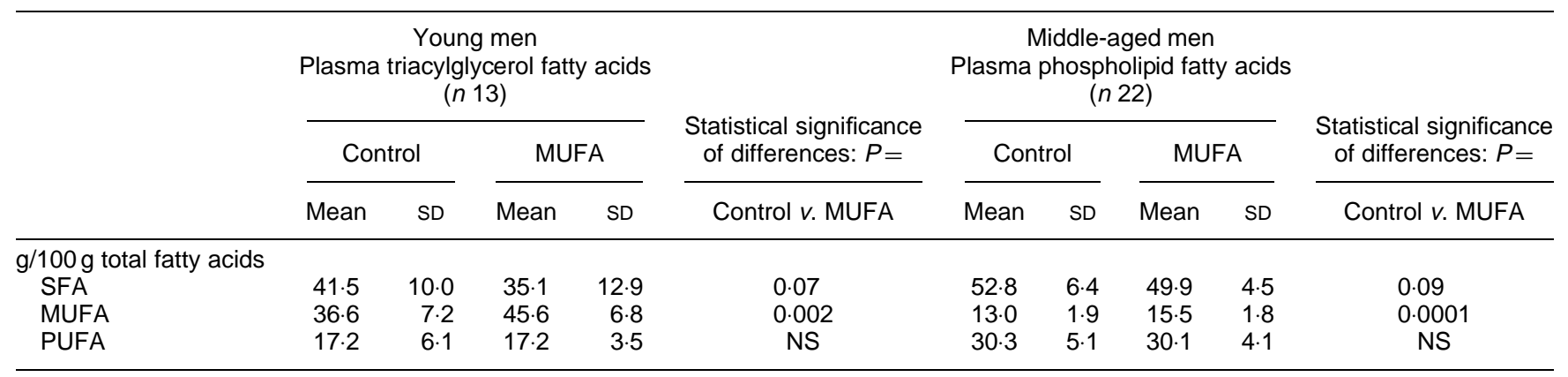

SFA, saturated fatty acids; PUFA, polyunsaturated fatty acids.

${ }^{*}$ For details of subjects and diets, see Table 2 and pp. 440-442. 


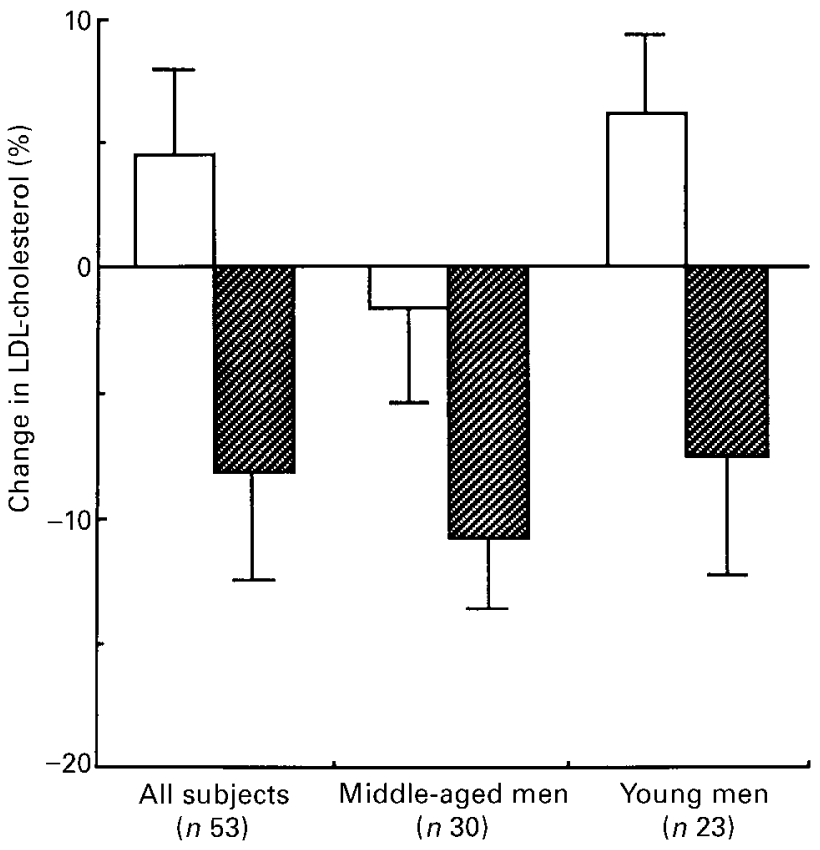

Fig. 1. Percentage changes in plasma LDL-cholesterol concentration in all subjects ( $n 53)$ and in middle-aged $(n 30)$ and young $(n 23)$ men from baseline to the end of an 8-week period of consuming a contro diet $(\square)$ or a monounsaturated fatty acid-enriched diet $(\square)$.

(SD 13) $\%$ and a decrease in LDL-cholesterol of $-7 \cdot 8$ (SD 20) $\%$ on the MUFA diet. The different responses of the two age groups to the two diets appears to be a reflection of their different baseline fasting LDL-cholesterol values since, as shown in Fig. 2, there was an inverse and significant

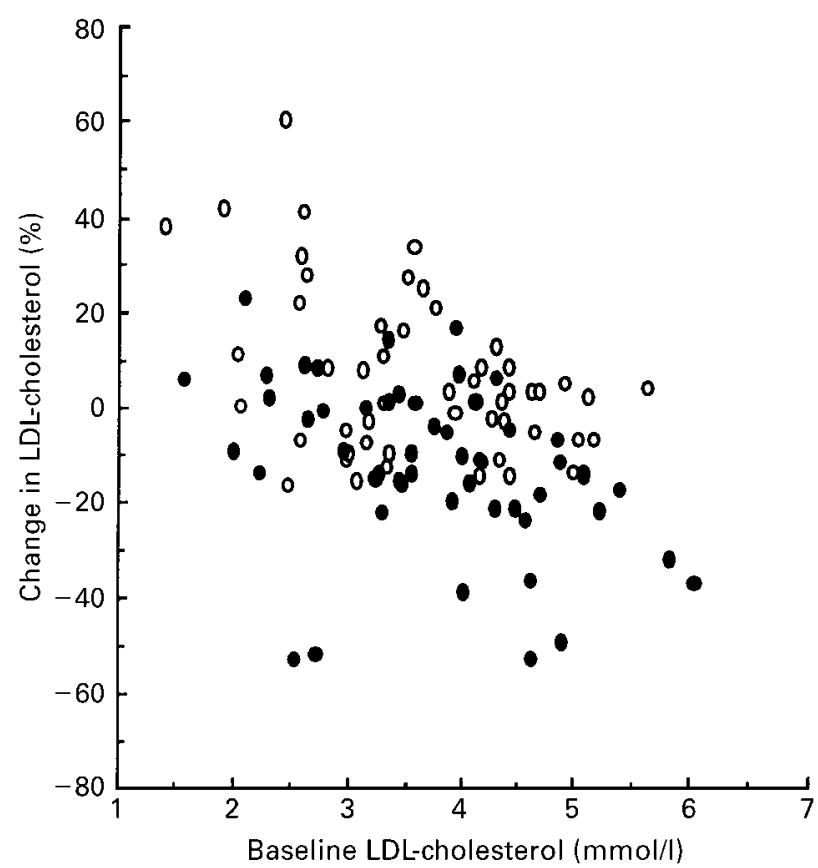

Fig. 2. Correlation between percentage change in plasma LDLcholesterol concentration and baseline LDL-cholesterol concentration $(\mathrm{mmol} / \mathrm{l})$ in fifty-three male subjects consuming a control diet $(O)$ or a monounsaturated fatty acid-enriched diet $(\bullet)$. 
correlation between baseline LDL-cholesterol levels and the percentage change in LDL-cholesterol from baseline to week 8 on each diet $(r-0.49 ; P<0.0005)$. The data indicate that subjects with the highest starting cholesterol concentrations showed the most marked reductions on the MUFA diet, whilst subjects with the lowest cholesterol concentrations showed the most marked increases on the control diet.

\section{Discussion}

As anticipated, the MUFA diet, which achieved a marked reduction in SFA intake to a mean level of 10.1 (SD 1.7) \% energy, achieved a significant reduction in LDL-cholesterol of $0.38 \mathrm{mmol} / \mathrm{l}$ in fifty-three healthy normocholesterolaemic young and middle-aged men. An important and novel aspect of the study was the use of typical manufactured foods as the intervention vehicles. Compliance with the diet was found to be excellent and the food products were of similar quality and palatability for both the control and MUFA arms of the study. The study used foods which were of similar quality and type to those available in UK and Irish supermarkets; this increased the acceptability of the diet and made it feasible to carry out a study which, although it used free-living subjects, was well controlled in terms of its single-blind design and achievement of the target fatty acid intakes, and highly acceptable with respect to the volunteers' lifestyles.

The mean difference in the levels of total cholesterol at the end of the control and the MUFA diets (0.29 (SD 0.61) $\mathrm{mmol} / \mathrm{l}$ ) was less than that which would be predicted by the Keys et al. (1957) equation $(0.39 \mathrm{mmol} / \mathrm{l})$, which uses the changes in dietary intake of SFA, PUFA and cholesterol to estimate the change in blood cholesterol. However the difference in LDL-cholesterol values between the two diets $(0.38$ (SD 0.64) $\mathrm{mmol} / \mathrm{l})$ was very close to that predicted by the Keys et al. (1957) equation. More recently Yu et al. (1995) have produced alternative equations which take into account the now well-accepted neutral effects of stearic acid on blood cholesterol levels. When this equation was applied to the diets used in the present study and the values for stearic acid intakes on the control and MUFA diets were used in the equation, the predicted changes in total cholesterol and LDL-cholesterol were found to be $0 \cdot 21$ and $0.11 \mathrm{mmol} / 1 \mathrm{respectively,} \mathrm{considerably} \mathrm{less} \mathrm{than} \mathrm{the}$ differences actually found. It is difficult from these comparisons to determine whether the addition of MUFA in the present diet added to the cholesterol-lowering effects of the reduced SFA intake. Comparison with the Keys et al. (1957) equation would suggest that the reduction in cholesterol reported here was entirely due to displacement of SFA from the diet, with no additional hypocholesterolaemic effect attributable to addition of MUFA, whereas comparison with the $\mathrm{Yu}$ et al. (1995) equation would suggest that the diet produced a reduction in cholesterol additional to that predicted from the changes in intakes of the cholesterolraising SFA.

Although the data presented here demonstrate a significant effect of a MUFA-rich diet on total and LDL-cholesterol concentrations, there was considerable individual variability in subjects' responses to the diet. Analysis of the data for middle-aged and young men separately showed the two groups to differ in their response to the two diets. In the middle-aged men there was a clear effect of the MUFA diet itself, with a mean reduction in LDL-cholesterol concentration of $-11 \%$, with no change in response to the control diet. In the young men, there was a mean increase in LDL-cholesterol concentration of $+6.2 \%$ on the control diet. In contrast there was a smaller reduction $(-7.8 \%)$ in LDL-cholesterol on the MUFA diet. Nevertheless the net effect of the two diets was similar in the two groups, with comparison of differences between end of the control diet and end of the MUFA diet producing similar findings, which was a net difference in cholesterol levels of 11-14\% between the two diets. A possible explanation for the different responses of the two groups of men to the control and MUFA diets was that the habitual diets of the two groups might have differed before the interventions, so that the responses to the control and MUFA diets varied according to baseline habitual diets. This was considered to be a plausible explanation since the young men each had a close family history of CHD and may have been more responsive to previous dietary advice concerning cholesterol reduction, thereby influencing their habitual fatty acid intakes. However, habitual dietary intakes assessed in the study by means of a $4 \mathrm{~d}$ diet diary, showed no significant differences in total fat or of the major fatty acid classes between the young and middle-aged men. It was noted, however, that young Irish men had lower intakes of SFA than either young or middle-aged UK men but the differences were not statistically significant. Caution must be applied in excluding differences in habitual diet as a possible explanation for these findings since there are major limitations in estimates of fatty acid intakes in free living populations due to the inadequate and outdated data available for fatty acid compositions in most food composition databases. Values for the complete fatty acid compositions of individual foods are limited to only a small proportion of the foods entered into the database and because of frequent changes in manufacturers' specifications, are known to be outdated even for major fat sources such as margarines and oils. Much greater confidence can be placed on the estimates of fatty acid intakes on the control and MUFA diets since the majority of foods used in the study, which represented $60 \%$ of total fat intake, were directly analysed for their fat contents and fatty acid compositions and this information was added to the database allowing more accurate estimation of true fat and fatty acid intakes. Nonintervention fat-containing foods included cheese and other dairy products, whose compositions are subject to less variability than other foods. Greater reliability could also be placed on portion sizes during the intervention study since pre-packaged foods were of standard size.

Another explanation for the different responses of young and middle-aged men may be that on the MUFA diet, the young men were achieving a greater proportion of their energy from fat and had a higher energy intake from SFA than did the middle-aged men. This may have mitigated against some of the benefits of the MUFA diet in this group. However the data shown in Fig. 2 suggest that the most likely explanation for the findings is that the effects of both diets are determined in part by the starting values for 
LDL-cholesterol, which are strongly associated with age. These data suggest that where baseline LDL-cholesterol level is elevated subjects are more likely to respond to a low-SFA-high-MUFA diet with a reduction in cholesterol levels, whereas the opposite is the case for subjects with modest or low cholesterol values. The data in Fig. 2 also illustrate the great heterogeneity in response to the two diets. Whilst variations in compliance may have contributed to this, other factors including genetic influences, need to be considered.

The public health considerations of the findings of the present study are worthy of some discussion. Law et al. (1994) have estimated that a $0.6 \mathrm{mmol} / \mathrm{l}$ reduction in LDLcholesterol level in middle-aged men would produce a corresponding decrease in the risk of CHD of $25-30 \%$. Based on these data the dietary changes achieved in the present study, if extrapolated to the middle-aged male population of the UK, would be expected to reduce risk of CHD by $16 \%$, with considerable benefit in terms of quality of life and cost to the health service. The 1994 report Nutritional Aspects of Cardiovascular Disease (Department of Health, 1994) concluded that reductions in plasma total and LDLcholesterol could best be achieved by a reduction in dietary SFA from the current level of intake of $16 \%$ energy to the desired level of $10 \%$ energy. The diet investigated in the present study achieved the same outcome, but without need for a reduction in the overall level of fat intake. The data presented here suggest that a high-MUFA diet offers a practical means of achieving target SFA intakes and of reducing plasma total and LDL-cholesterol concentrations, and may be more acceptable to some consumers than advice to reduce fat intakes to levels less than $33 \%$ of dietary energy. The method used to achieve target dietary intakes in the present study is one which would be readily achievable if sufficient products were available in food retail outlets. No technological or other problems were encountered in producing the MUFA-enriched versions of the commercially available products, although some modifications might be needed to achieve a more comprehensive range of items.

The present diet may be of particular value in children and adolescents in whom fat and energy restriction may be unnecessary, but in whom attempts to achieve and sustain low circulating cholesterol levels could have major benefits in later life. In overweight and obese adults, low-fat diets must continue to be advised, since energy restriction remains the focus of attention in these groups.

\section{Acknowledgements}

This study was supported by a grant from the Ministry of Agriculture, Fisheries and Food and in part by the European Union. The authors acknowledge the invaluable contribution of Van den Bergh UK Ltd in proving fats and spreads for the study and Sainsburys UK Ltd in providing contact with food manufacturers who prepared the readymade foods. The technical assistance of Emma Copeland, Efthikos Pentaris, Nigel Purlonger and Judith Wilson is gratefully acknowledged.

\section{References}

Department of Health (1991) Dietary Reference Values for Food Energy and Nutrients for the United Kingdom. Report on Health and Social Subjects no. 41. London: H. M. Stationery Office.

Department of Health (1994) Nutritional Aspects of Cardiovascular Disease. Report on Health and Social Subjects no. 46. London: H. M. Stationery Office.

Friedewald WT, Levy RI \& Fredrickson DS (1972) Estimation of the concentration of low density lipoprotein cholesterol in plasma without use of preparative ultracentrifugation. Clinical Chemistry 18, 499-502.

Gregory J, Foster K, Tyler H \& Wiseman M (1990) The Dietary and Nutritional Survey of British Adults. London: H. M. Stationery Office.

Keys A, Anderson JT \& Grande P (1957) Prediction of serumcholesterol responses of man to changes in fats in the diet. Lancet ii, 959-966.

Law MR, Wald NJ \& Thompson SG (1994) By how much and how quickly does reduction in serum cholesterol concentration lower risk of ischaemic heart disease? British Medical Journal 308, 367-372.

Ministry of Agriculture, Fisheries and Food (1992) Household Food Consumption and Expenditure. London: H. M. Stationery Office.

Pyörälä K, De Backer G \& Graham L (1994) Prevention of coronary heart disease in clinical practice, recommendations of the Task Force of the European Society of Cardiology, European Atherosclerosis Society and European Society of Hypertension. European Heart Journal 15, 1300-1331.

Roche HM, Zampelas A, Knapper JME, Webb D, Brooks C, Jackson KG, Wright JW, Gould BJ, Kafatos A, Gibney MJ \& Williams CM (1998) The effect of chronic olive oil dietary intervention on acute postprandial triacylglycerol and factor VII metabolism. American Journal of Clinical Nutrition 68, 552560.

Scandinavian Simvastatin Survival Group (1994) Randomised trial of cholesterol lowering in 4444 patients with coronary heart disease, the Scandinavian Simvastatin Survival Study (4S). Lancet 344, 1383-1389.

Shepherd J, Stuart MC, Ford I, Isles C, Lorimer AR, Macfarlane PW, McKillop JH \& Packard CJ (1995) Prevention of coronary heart disease with pravastatin in men with hypercholesterolemia. New England Journal of Medicine 333, 1301-1307.

Yaqoob P, Knapper JA, Webb DH, Williams CM, Newsholme EA \& Calder PC (1998) Effect of olive oil on immune function in middle aged men. American Journal of Clinical Nutrition 67, 129-135.

Yu S, Derr J, Etherton TD \& Kris-Etherton PM (1995) Plasma cholesterol-predictive equations demonstrate that stearic acid is neutral and monounsaturated fatty acids are hypocholesterolemic. American Journal of Clinical Nutrition 61, 11291139. 